\title{
Overview of the background reduction techniques applied in the SoLid experiment
}

\author{
lanthe Michiels*t \\ Universiteit Gent \\ E-mail: ianthe.michielseugent.be
}

\begin{abstract}
The SoLid experiment - short for "Search for oscillations with a ${ }^{6}$ Lithium detector" - is designed to investigate reactor antineutrino oscillations at a very short baseline of 5.5 to $10 \mathrm{~m}$. Its aim is to confirm or reject the light sterile neutrino hypothesis [1], as well as to perform a precise ${ }^{235} \mathrm{U}$ spectrum measurement. In the winter of 2014-2015 a large scale detector prototype of the SoLid experiment was commissioned and installed at the BR2 reactor site of the SCK•CEN in Mol, Belgium. These proceedings discuss the analysis of the data taken with this detector prototype, mainly focussing on background reduction techniques. The correlated and accidental backgrounds are shown to be reduced respectively by a factor of $\mathscr{O}(10)$ and $\mathscr{O}(100)$. Currently, machine learning techniques are being investigated to further improve the signal-to-noise ratio.
\end{abstract}

38th International Conference on High Energy Physics 3-10 August 2016

Chicago, USA

\footnotetext{
${ }^{*}$ Speaker.

${ }^{\dagger}$ for the SoLid collaboration
} 


\section{Introduction}

To this day, the neutrino sector remains one of the most intriguing topics within the field of experimental particle physics. Although the 3-neutrino oscillation model was confirmed by numerous experiments and has been rewarded with the Nobel Prize in 2015 [2], some persistent anomalies in measured neutrino oscillation patterns remain. These anomalies have lead to a hypothesis suggesting the existence of a fourth, light sterile neutrino, with parameters around $\Delta m^{2} \sim 1 \mathrm{eV}^{2}$ and $\sin ^{2}(2 \theta) \sim 0.1$. Currently, various experiments aim to explore the sterile neutrino hypothesis, one of which is the SoLid experiment located at the SCK•CEN in Mol, Belgium.

These proceedings will discuss the analysis of the data taken with a first large scale detector module of the SoLid experiment, mainly focussing on background reduction techniques. A more general discussion on the SoLid experiment, its detector design and the construction can be found in Ref. [3].

\section{SoLid in a nutshell}

The SoLid experiment - short for "Search for oscillations with a ${ }^{6}$ Lithium detector" - will investigate reactor antineutrino oscillations at a very short baseline of 5.5 to $10 \mathrm{~m}$. Its aim is to confirm or reject the sterile neutrino hypothesis, as well as to perform a precise ${ }^{235} \mathrm{U}$ spectrum measurement. For this, the experiment uses a new segmented detector technology based on small cubes of polyvinyl-toluene (PVT) scintillator for the detection of electromagnetic (EM) interactions and thin sheets of ${ }^{6} \mathrm{LiF}: \mathrm{ZnS}(\mathrm{Ag})$ for neutron capture. Figure 1 illustrates how a reactor antineutrino interacts in part of the detector volume, resulting in an inverse beta decay (IBD) reaction in which a positron and a neutron are created. The positron annihilates in the PVT, giving a fast and sharp scintillation pulse; the neutron, however, needs some time to thermalize, before it can be captured by the ${ }^{6} \mathrm{Li}$. The combination of the prompt positron signal and a delayed series of decay pulses from the ${ }^{6} \mathrm{LiF}: \mathrm{ZnS}$ mixture builds up the antineutrino signature. For more details on the SoLid detection principle, one can consult Ref. [4].

In the winter of 2014-2015 a large scale detector prototype, called SM1, was commissioned and installed at the reactor site in Mol. The SM1 detector consists of 9 vertical planes, each filled with 256 PVT cubes of $(5 \times 5 \times 5)$ $\mathrm{cm}^{3}$, resulting in a total weight of $288 \mathrm{~kg}$.

\section{Experimental backgrounds}

The experimental backgrounds present at a nuclear reactor site, can be divided into two

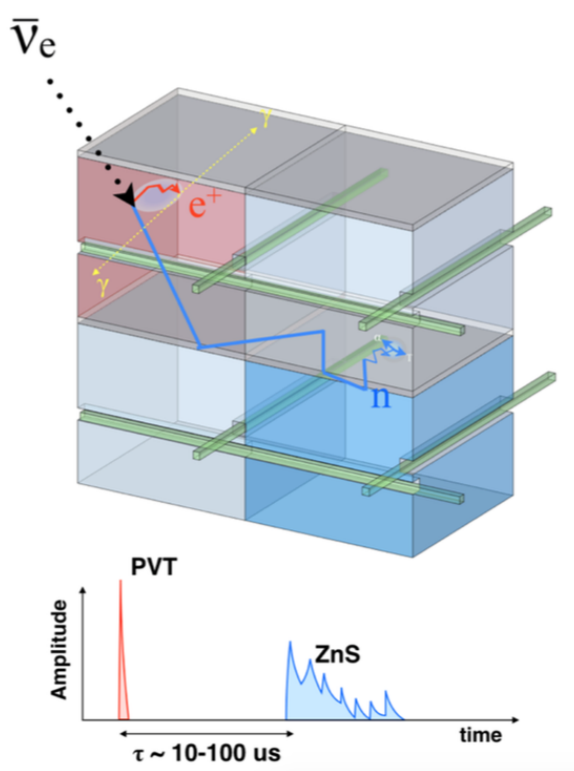

Figure 1: Schematic view of an antineutrino interaction with part of the SoLid detector (top). The resulting IBD signature is also sketched (bottom). 
types; accidental and correlated backgrounds.

The former originate from a random coincidence of an EM like signal and a neutron like signal, which can be created by reactor induced gammas and neutrons. The latter come from time correlated EM and neutron events, which are thought to be related to spallation neutrons from cosmic muons or cosmic high energy neutrons. Also radioactively induced neutrons contribute to the time correlated category, e.g. from decay chains of Bi/Po that contaminates the detector material.

\section{Background reduction techniques}

The accidental background can easily be studied by using shifted time windows, randomly combining EM with neutron signals. It is found that this type of background is significantly reduced when a lower energy threshold is applied to the EM signal and a limitation is placed on the distance $\Delta r$ between the neutron and positron signals. Figure 2 illustrates the power of the SoLid detector's segmentation in providing direct methods for discriminating signal from background.

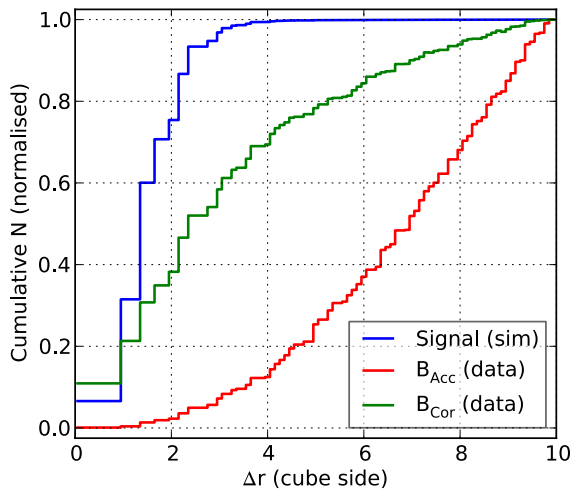

Figure 2: The high segmentation of the SoLid detector enables the use of IBD event topology as a discrimination against backgrounds.

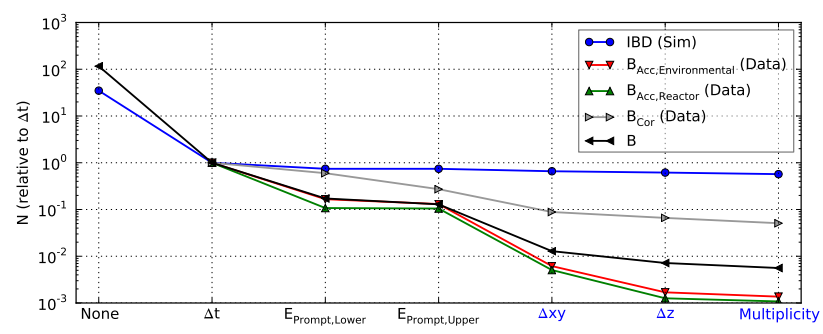

Figure 3: The relative rate reduction of the backgrounds and simulated signal by sequential rectangular cuts for the SoLid experiment. Because of its larger overlap with the IBD signal, the correlated background is harder to eliminate and each contribution to this type of background and its characteristics have to be understood individually. Muon identification is used to veto the contribution of muon induced events and a multiplicity cut on the number of EM signals can be effective to reduce fast neutron induced proton recoil events. In figure 3 the reduction of the different kinds of background as a function of the applied cuts is shown.

In particular the accidental backgrounds are shown to be significantly reduced; the full series of cuts lowers them by a factor of 200. Since a higher signal-to-noise ratio allows for increased sensitivity in the oscillation search, more advanced background reduction techniques have been investigated.

As a first step, an IBD analysis based on combined likelihoods was performed. Here, a new parameter was constructed for each event, based on a combination of time and spatial properties of the signals. For a given pair of a neutron and an EM candidate with specific values of $\Delta T, \Delta R, \Delta X$, 
$\Delta Y, \Delta Z, \ldots$ a new likelihood parameter is calculated, called the Global Likelihood (GL):

$$
G L=\frac{L_{\text {sim }}}{L_{\text {uncorr }}+L_{\text {corr }}+L_{\text {sim }}}
$$

with

$$
\begin{aligned}
& L_{\text {sim }}=f_{\Delta x}^{\text {sim }}(\Delta X) \times f_{\Delta y}^{\text {sim }}(\Delta Y) \times f_{\Delta z}^{\text {sim }}(\Delta Z) \times f_{\Delta t}^{\text {sim }}(\Delta T), \\
& L_{\text {uncorr }}=f_{\Delta x}^{\text {uncorr }}(\Delta X) \times f_{\Delta y}^{\text {uncorr }}(\Delta Y) \times f_{\Delta z}^{\text {uncorr }}(\Delta Z) \times f_{\Delta t}^{\text {uncorr }}(\Delta T), \\
& L_{\text {corr }}=f_{\Delta x}^{\text {corr }}(\Delta X) \times f_{\Delta y}^{\text {corr }}(\Delta Y) \times f_{\Delta z}^{\text {corr }}(\Delta Z) \times f_{\Delta t}^{\text {corr }}(\Delta T) .
\end{aligned}
$$

The distribution of the $G L$ parameter is shown in figure 4 for different sets of data; the signal distribution is based on simulated events, the background distributions are constructed from data. It was found that this Likelihood Discriminator can further reduce the backgrounds by $\sim 30 \%$, while retaining the same signal efficiency as the cut-based studies.

\section{Outlook}

The data taking process with the SM1 prototype has given valuable insights in the reconstruction and treatment of experimental backgrounds. The collaboration has developed various background reduction methods and was able to check these with both data and Monte Carlo simulations. The correlated backgrounds were reduced by a factor of 20 and the contribution of accidental backgrounds was lowered by a factor of 200. Currently, machine learning techniques such as Boosted Decision Trees (BDT) and Support Vector Machines (SVM) are being investigated and they show that even better

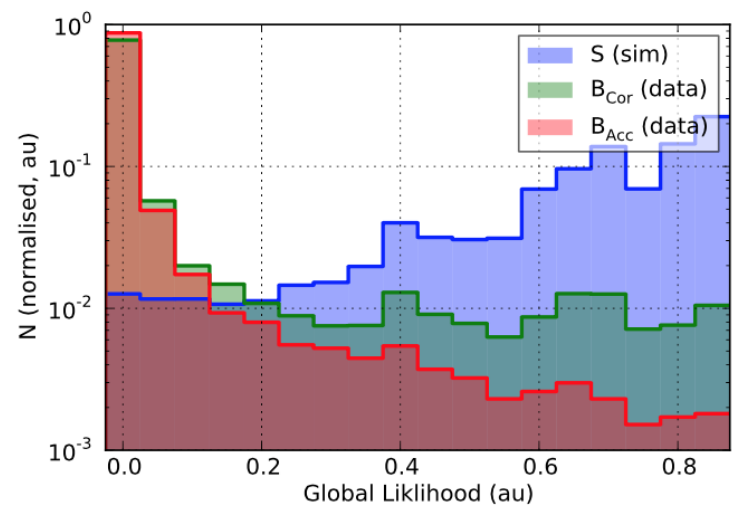

Figure 4: The global likelihood distributions for the simulated IBD signal and background data sets. background reduction is possible.

\section{References}

[1] K. N. Abazajian et al., Light sterile neutrinos: A white paper, arXiv:1204.5379

[2] The Royal Swedish Academy of Sciences, Nobel Prize in Physics 2015, nobelprize.org

[3] C. Moortgat, SoLid: why and how we search for sterile neutrinos, these proceedings

[4] L. N. Kalousis, SoLid: A compact neutrino detector for very short-baseline neutrino experiments, these proceedings 